\title{
Deregulation of Nicotinamide N-Methyltransferase and Gap Junction Protein Alpha-1 Causes Metastasis in Adenoid Cystic Carcinoma
}

\author{
KANA ISHIBASHI ${ }^{1}$, KOTARO ISHII ${ }^{1}$, GORO SUGIYAMA ${ }^{1}$, TOMOKI SUMIDA ${ }^{1}$, TSUYOSHI SUGIURA ${ }^{2}$, \\ YU KAMATA ${ }^{1}$, KATSUHIRO SEKI ${ }^{1}$, TAKAHIRO FUJINAGA ${ }^{1}$, WATARU KUMAMARU ${ }^{1}$, YOSUKE KOBAYASHI ${ }^{1}$, \\ NAOMI HIYAKE ${ }^{1}$, HIROYUKI NAKANO ${ }^{1}$, TOMOHIRO YAMADA ${ }^{1}$ and YOSHIHIDE MORI ${ }^{1}$ \\ ${ }^{1}$ Section of Oral \& Maxillofacial Surgery, Division of Maxillofacial Diagnostic and Surgical Sciences, \\ Faculty of Dental Science, Kyushu University, Fukuoka, Japan; \\ ${ }^{2}$ Department of Maxillofacial Diagnostic and Surgical Science, Field of Oral and Maxillofacial Rehabilitation, \\ Graduate School of Dental Science, Kagoshima University, Kagoshima, Japan
}

\begin{abstract}
Background/Aim: Adenoid cystic carcinoma $(A d C C)$ is a malignant tumor that occurs in the salivary glands and frequently metastasizes. The aim of this study was to identify factors mediating AdCC metastasis. Materials and Methods: We established three AdCC cell lines by orthotropic transplantation and in vivo selection: parental, highly metastatic (ACCS-M-GFP), and lymph node metastatic (ACCS-LN-GFP) cells. Results: We examined the three cell lines. DNA microarray indicated significantly altered processes in ACCS-LN-GFP cells: particularly, the expression of nicotinamide $N$-methyltransferase (NNMT) was enhanced the most. NNMT is associated with tumorigenesis and is a potential tumor biomarker. Concomitantly, we foundsignificant down-regulation of gap junction protein alpha-1. We suggest that ACCS-LN-GFP cells acquire cancer stem cell features involving the up-regulation of NNMT and the loss of gap junction protein alpha-1, leading to epithelialmesenchymal transition and consequent AdCC metastasis. Conclusion: NNMT is a potential biomarker of AdCC.
\end{abstract}

Adenoid cystic carcinoma (AdCC) is a common cause of head and neck malignant tumors. AdCC is characterized by slow development, local invasion of the nerves and vessels,

Correspondence to: Dr. Kotaro Ishii, Section of Oral \& Maxillofacial Surgery, Division of Maxillofacial Diagnostic and Surgical Sciences, Faculty of Dental Science, Kyushu University, 3-1-1, Maidashi, Higashi-ku, Fukuoka, 812-8582, Japan. Tel: +81 926426452, Fax: +81926426392, e-mail: kishii17@dent.kyushu-u.ac.jp

Key Words: Adenoid cystic carcinoma, primary tumor cell lines, metastatic cell lines, epithelial-mesenchymal transition, nicotinamide $\mathrm{N}$-methyltransferase. and distant metastases, especially in the lungs. Approximately $40-60 \%$ of AdCCs metastasize $(1,2)$ and the metastases are characterized by low sensitivity for chemotherapy or radiotherapy; therefore, $\mathrm{AdCC}$ is often difficult to treat, and associated with poor prognosis (3). Furthermore, molecular targeted therapies have not been developed to treat this disease, as it has happened for other malignant tumors (4). It is crucial to elucidate the biological characteristics of AdCC with respect to tumorigenesis and tumor metastasis. To understand the mechanism of AdCC metastasis, we have previously established two AdCC cell lines green fluorescent protein (GFP) gene transferred by injecting cells to the tongue and in vivo selection in nude mice: the parental ACCS-GFP and the highly metastatic ACCS-M-GFP cell lines. DNA microarray analysis was examined and the results revealed significantly altered biological molecules associated to cell adhesion and signaling in ACCS-M-GFP cells. In particular, a significant down-regulation of cell adhesion molecules such as E-cadherin and integrin and up-regulation in the expression of vimentin was observed. We concluded that the epithelialmesenchymal transition (EMT) is the important phenomenon that cells disseminate from the primary tumor site and lead to AdCC metastasis (5).

In a different study, we confirmed a direct relation between the EMT and cancer stem-like cells (CSCs) in the highly metastatic ACCS-M-GFP cells. Furthermore, we reported that the T-box transcription factor Brachyury, which is also a marker of mesoderm differentiation, regulates CSCs and the EMT in AdCC (6). Brachyury-knockdown exerted stronger effects on cancer stemness and the EMT phenotype than the knockdown of the conventional CSCs regulator Sox2. By reducing the stemness of CSCs, Brachyury knockdown significantly inhibited tumorigenicity and metastasis in vivo (7). 
In our previous studies, we used cell lines established from the primary tumor site, with high metastatic potential. However, to identify metastasis-associated molecules it is important to examine cells that have metastasized through the detachment from the tumor mass, intra- and extravascular invasion and tumor formation at distant sites. Therefore, in this study, we established the lymph node metastatic cell line ACCS-LN-GFP from AdCC cells, using orthotropic transplantation in nude mice. The derived lymph node metastatic and parental cell lines were then subjected to DNA microarray analysis. Data mining analysis identified uniquely expressed genes in lymph node metastatic cells, and this result was validated using ACCS cell lines. Both the computational and experimental validation highlighted the biological alterations associated with lymph node metastatic ACCS cells.

\section{Materials and Methods}

Cells and culture. The human adenoid cystic carcinoma cell lines ACCS, ACCS-GFP, and ACCS-M-GFP were established in our laboratory, as reported previously $(5,8)$. The parental ACCS cells and GFP-transfected ACCS (ACCS-GFP) cells displayed similar morphologies, growth rates, and tumorigenicity both in vitro and in vivo. As the parental ACCS, the tumorigenicity of the ACCS-GFP cells was low (22.2\% incidence). We examined tumor formation in the tongue of nude mice injected with ACCSGFP cells and observed, under excitation light, the fluorescence associated with the tumors. We performed in vivo selection of the clones with higher tumorigenicity by repeatedly recovering the tumor cells and transplanting them into the tongue of nude mice. Through this process, we obtained ACCS-M-GFP cells, a sub-line exhibiting high tumorigenicity ( $100 \%$ incidence) and a high frequency of spontaneous metastasis to submandibular lymph nodes (100\% incidence). The histological and immunohistochemical features of ACCS-M-GFP tumors were similar to those of the solid AdCC. These cell lines were maintained as monolayer cultures in Dulbecco's modified Eagle's medium (DMEM; Sigma-Aldrich, St. Louis, MO, USA) supplemented with $10 \%$ fetal bovine serum (FBS; Filton Pty, Brooklyn, Australia), 2 mM L-glutamine (Wako Pure Chemical Industries, Osaka, Japan), penicillin G (Meiji Seika Pharma, Tokyo, Japan), and streptomycin (Meiji Seika Pharma, Tokyo, Japan) in a $5 \% \mathrm{CO}_{2}$ incubator at $37^{\circ} \mathrm{C}$. The cell lines were passaged using $0.05 \%$ trypsin/EDTA (Gibco, Grand Island, NY, USA) and phosphate-buffered saline (PBS).

Animals and ACCS-M-GFP metastatic orthotropic implantation mouse model. The animal experimental protocols were approved by the Animal Care and Use Committee of the Kyushu University, (Fukuoka, Japan). Eight-week-old female athymic nude mice (BALBcAJcl-nu) were purchased from Kyudo (Fukuoka, Japan). The mice were housed in laminar flow cabinets under specific pathogen-free conditions, in facilities approved by the Kyushu University. For the experimental metastasis studies, $1 \times 10^{6}$ cells in $40 \mu \mathrm{l}$ PBS were injected into the tongue of mice under intraperitoneal diethyl ether anesthesia, using a syringe with a 27gauge disposable needle (TOP Plastic Syringe, Tokyo, Japan). The primary tumor volumes were measured weekly, calculated as length $x$ width $\times$ thickness, and the mice were sacrificed when the primary tumor volume reached $100 \mathrm{~mm}^{3}$. After sacrifice, the tongue, cervical lymph nodes, lungs, and liver were observed macroscopically. The tumors and the metastasis of GFP-transfected clones were also visualized macroscopically under light excitation. After visualization, the primary tumors and metastatic sites were examined pathologically and immunohistochemically. Lymph metastatic AdCC tumors were cultured on plastic tissue culture dishes using the explant cell culture method.

Cell count and cell growth curves for the AdCC cell lines. Subconfluent cells were observed under a fluorescence microscope (BZ8000; Keyence, Osaka, Japan), and the cells in four fields were counted. Cell growth was evaluated counting the ACCS-GFP, ACCS-M-GFP, and ACCS-LN-GFP cells cultured on 3-cm-diameter plastic plates, every $24 \mathrm{~h}$. Assays were performed in triplicate and repeated three times.

Wound healing assay. Each cell line $\left(3 \times 10^{5}\right.$ cells $)$ was seeded on a 6 -well plate, and $24 \mathrm{~h}$ later 'wounds' were created by scratching the plate surface with a $200-\mu l$ pipette tip. The plate was then washed with medium, and observed under a fluorescence microscope. The wound regions were photographed after 8,16 , and $24 \mathrm{~h}$. The wound area was calculated using the following formula: Wound area (\% of control) $=$ Wound area at the indicated period $\times 100 /$ initial wound area. All experiments were carried out in triplicate and repeated three times

Total RNA isolation. Total RNA was isolated from ACCS-GFP, ACCS-M-GFP, and ACCS-LN-GFP cells using TRIzol Reagent (Invitrogen, Carlsbad, CA, USA) and was purified using an SV Total RNA Isolation System (Promega, Madison, WI, USA) according to the manufacturer's instructions. RNA samples were quantified with an ND-1000 spectrophotometer (Nano Drop Technologies, Wilmington, DE, USA), and their quality was confirmed with an Experion System (Bio-Rad Laboratories, Hercules, CA, USA).

Gene expression microarrays. The cRNA was amplified, labeled, and hybridized to a $60 \mathrm{~K}$ Agilent 60-mer oligomicroarray (SurePrint G3 Human Gene Expression Microarray $8 \times 60 \mathrm{~K}$, v2; Agilent Technologies, Santa Clara, CA, USA) according to the manufacturer's instructions. All hybridized microarray slides were scanned by an Agilent scanner. Relative hybridization intensities and background hybridization values were calculated using the Agilent Feature Extraction software (9.5.1.1).

Data analysis and filter criteria. Raw signal intensities and flags for each probe were calculated from hybridization intensities (gProcessedSignal), and spot information (e.g., gIsSaturated) according to the procedures recommended by Agilent. Following are the flag criteria in the GeneSpring Software: Absent (A), 'Feature is not positive and significant' and 'Feature is not above background'; Marginal (M), 'Feature is not uniform', 'Feature is saturated', and 'Feature is a population outlier'; and Present $(\mathrm{P})$ : others. The raw signal intensities of two samples were $\log 2-$ transformed and normalized by quantile algorithm with the 'preprocessCore' library package by using the open source Bioconductor software. 
We selected probes that we called the ' $\mathrm{P}$ ' flag in both samples. To identify up- and down-regulated genes, we calculated the Zscores and ratios (non-log scaled fold-change) from the normalized signal intensities of each probe for comparison between control and experiment samples. Then, we established the criteria for regulated genes: up-regulated genes, Z-score $\geq 2.0$ and ratio $\geq 5$-fold; downregulated genes, $Z$-score $\leq-2.0$ and ratio $\leq 0.2$. The extracted data were analyzed using DAVID, a database of the National Institute of Allergy and Infectious Diseases (NIAID). In addition, microarray data analysis was supported by Cell Innovation platform.

Quantitative PCR $(q P C R)$. The expression of the nicotinamide Nmethyltransferase (NNMT) gene in AdCC cells was quantified by qPCR. Total RNA was extracted from ACCS-M-GFP and ACCSLN-GFP cells using an RNeasy Mini kit (Qiagen, Chatsworth, CA, USA) and used for first-strand cDNA synthesis. The mRNA levels were quantified in triplicate using a qPCR system (Roche Diagnostics, Mannheim, Germany) with the Light Cycler ${ }^{\circledR}$ Fast Start DNA Master SYBR Green I Kit (Roche Diagnostics, Mannheim, Germany). The specific NNMT primers were (F) 5'GAGCAGAAGTTCTCCAGCCT-3' and (R) 5'-ACCATTCG ATTGTGTAGCCA-3'. Amplification was performed using the following conditions: denaturation at $95^{\circ} \mathrm{C}$ for $10 \mathrm{~min}$ followed by 46 cycles of annealing at $60^{\circ} \mathrm{C}$ for $10 \mathrm{sec}$ and extension at $72^{\circ} \mathrm{C}$ for $10 \mathrm{sec}$. Dissociation curve analyses confirmed that the signals corresponded to unique amplicons. Each experiment was done in triplicate, and the results were normalized against the mRNA levels of glyceraldehyde-3-phosphate dehydrogenase $(G A P D H)$ obtained from parallel assays and analyzed using the LightCycler ${ }^{\circledR} 2.0$ System software (Roche Diagnostics, Mannheim, Germany).

Western blot. Cultured cells were rinsed with PBS and then lysed by sonication in sodium dodecyl sulfate (SDS) lysis buffer (50 mM Tris$\mathrm{HCl}$ [pH 6.8], 2\% SDS, 10\% glycerol, and 6\% mercaptoethanol) containing a protease inhibitor cocktail (Sigma-Aldrich, St. Louis, MO, USA). The protein contents of the lysates and fractionated samples were quantified using the Bio-Rad Protein Assay Kit (BioRad Laboratories, Hercules, CA, USA). An equal amount of protein $(30 \mu \mathrm{g})$ from each sample was electrophoresed on $10 \%$ SDS polyacrylamide gels and transferred electrophoretically onto a nitrocellulose membrane (Bio-Rad Laboratories, Hercules, CA, USA) After washing with TBST (25 mM Tris- $\mathrm{HCl}$ [pH 8.2], $144 \mathrm{mM} \mathrm{NaCl}$, and $0.1 \%$ Tween 20 ), the membranes were blocked with $5 \%$ non-fat skimmed milk in TBST at $20-25^{\circ} \mathrm{C}$. Immunoreactive bands were visualized using horseradish peroxidase-conjugated secondary antibodies (DAKO, Carpinteria, CA, USA) and ECL detection reagents (Amersham Pharmacia Biotech, Piscataway, NJ, USA). Bands were quantified using the Quantity One Software (Bio-Rad Laboratories, Hercules, CA, USA) after scanning by computerassisted densitometry (ChemiDoc XRS-J; Bio-Rad Laboratories, Hercules, CA, USA). Beta-actin was used as a loading control. The primary antibodies used for immunoblotting were: mouse monoclonal antibody against NNMT (ab119758, Abcam, Cambridge, UK); and mouse monoclonal antibody against $\beta$-actin (A5316, Sigma-Aldrich, St. Louis, MO, USA).

Statistical analysis. All data are presented as mean \pm the standard deviation (SD), as analyzed via analysis of variance and Student's $t$-test, and processed using the SPSS 13.0 software (IBM, Armonk, NY, USA). Statistical significance was defined as $p<0.05$.

\section{Results}

Establishment of the lymph node metastatic cell line ACCS$L N-G F P$. We previously created the ACCS-GFP cell line, upon stable GFP transfection in AdCCs (5). ACCS-GFP cells implanted in the tongue of nude mice showed low tumorigenicity and no metastasis formation. We repeatedly injected these cells in the mouse tongue and isolated the tumors that originated. As a result of this multi-step selection, we obtained ACCS-M-GFP cells, a cell line with $100 \%$ rate of metastasis. The tumor primary site and the metastatic sites (under an optic (Figure 1A, D and G) were visualized as green spots under a fluorescence microscope (Figure 1B, E and H). Histopathological examination of the primary tumor (Figure 1C) indicated that its border was unclear and that large and small cancer nests were scattered. On the other hand, the pathological examination of the cervical lymph node metastases indicated that the cancer had undergone follicular-shaped proliferation (Figure 1F). Additionally, the tongue tumors formed upon injection of ACCS-LN-GFP cells showed the solid pattern of AdCC (Figure 1I). We then successfully established the lymph node metastatic cell line from the lymph node metastatic tumors. We also attempted establishing a lung metastatic cell line with the same procedure. However, we were not successful, because the lung metastases were too small. In this study, we used the three cell lines: ACCS-GFP, ACCS-M-GFP, and ACCS-LN-GFP.

Morphology of ACCS-GFP and ACCS-LN-GFP cells. ACCSGFP and ACCS-LN-GFP cell images were obtained using a phase-contrast microscope. ACCS-GFP cells exhibited a thin, small crescent shape, whereas ACCS-LN-GFP cells were oval, with swollen cytoplasm and nucleus (Figure 2A). The cellular formation of ACCS-M-GFP is equal to that of ACCSLN-GFP (data not shown). Additionally, the number of ACCS-LN-GFP cells in an optical field was approximately 2.5 times lower than that of ACCS-GFP cells (Figure 2B).

Proliferation of the ACCS cell lines. The proliferative ability of the ACCS cell lines was evaluated. ACCS-GFP cells proliferated 1.2 times faster than ACCS-M-GFP or ACCSLN-GFP cells at 6 days, at which point all cell lines reached confluency (Figure 3).

Migration of the ACCS cell lines. The migratory ability of the ACCS cell lines was examined in a wound healing assay (Figure 4). The wound area in the ACCS-GFP cell culture after $24 \mathrm{~h}$ from the scratch was reduced by $\sim 50 \%$. Notably, at the same time point, the wound areas of the ACCS-MGFP and ACCS-LN-GFP cell cultures were $\sim 40 \%$ and $~ 30 \%$ of the original ones, respectively. These data indicated that the migratory ability of the ACCS-LN-GFP cells had a 

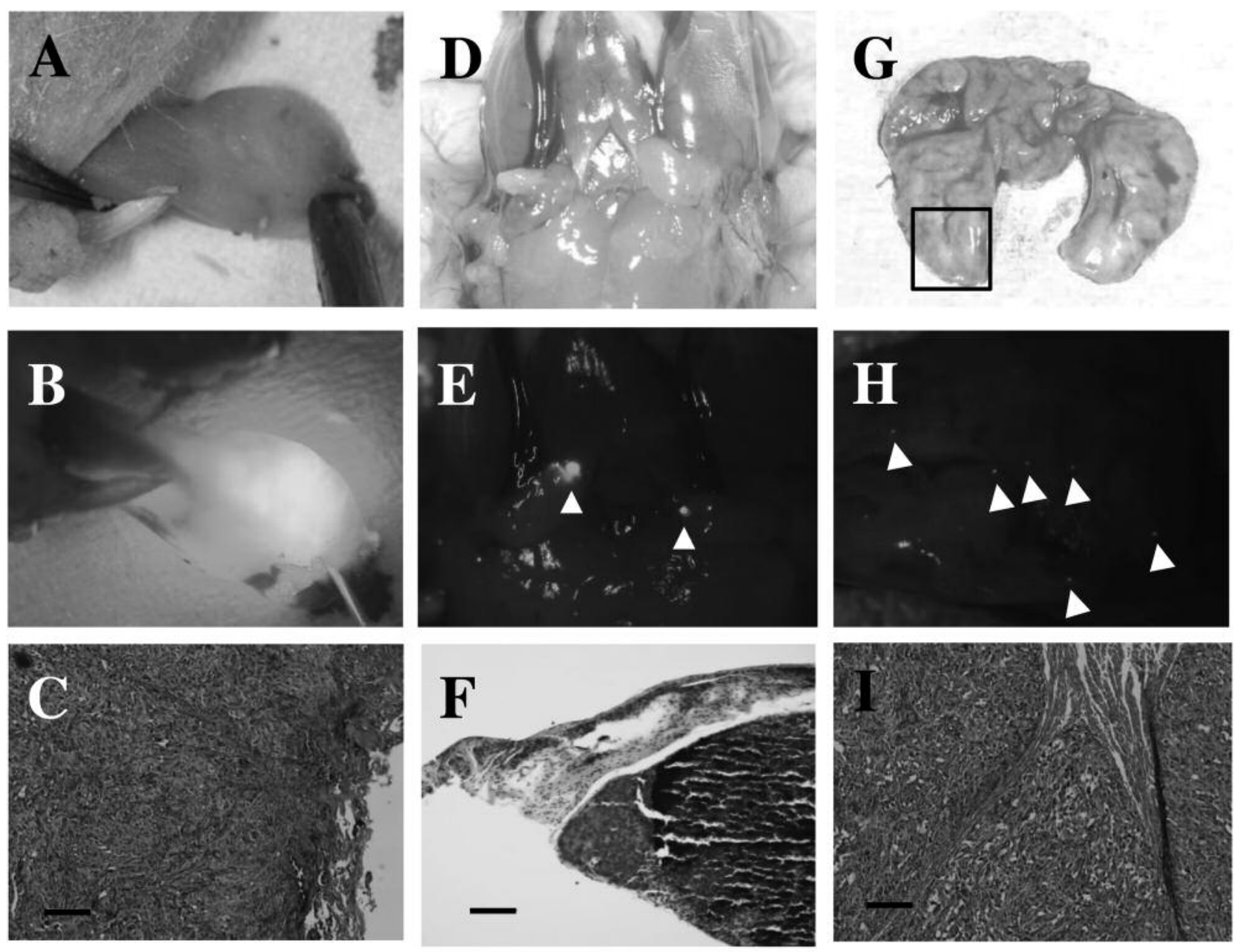

Figure 1. Detection of tumors and metastases upon ACCS-M-GFP injection. (A and B) Tongues, $(D$ and E) lymph nodes, and $(G$ and $H)$ lungs of nude mice injected with ACCS-M GFP cells were examined to detect tumors or metastases. (A, D, and G) Photographs captured under normal light and (B, $E$, and $H$ ) a fluorescence microscope are shown. The sites of lung metastasis are indicated by arrowheads. Micro-metastasis (GFP-positive) were observed in the lymph nodes and lungs. (C) Histopathology of primary tumors, (F) metastatic lymph nodes, and (I) tumor detected after ACCS-LN-GFP cells were injected into mouse tongue. After the mice were sacrificed, the tongue and the metastatic lymph nodes were removed and fixed with $2 \%$ paraformaldehyde

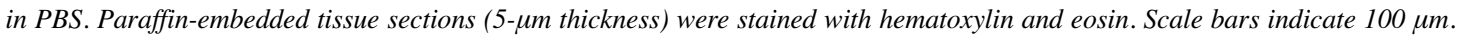

tendency of the increment compared to ACCS-GFP cells. However, there was no significant difference among them.

Microarray analysis. We previously reported the microarray analysis of ACCS-GFP and ACCS-M-GFP cells (5). Similarly, we continued our investigation by performing DNA microarray analysis using the ACCS-M-GFP and ACCS-LN-GFP cells, to uncover their differences at the molecular level. The results allowed us to comprehensively analyze the changes in ACCS-M-GFP and ACCS-LN-GFP gene expression, using 49,178 gene probes to search for metastasis-related genes in the two cell lines. A scatter plot of the signal values of ACCS-M-GFP and ACCS-LN-GFP gene expression showed the data with little dispersion and high reliability. Genetic differences were recognized when expression increased, with a $Z$-score $\geq 2.0$ and a ratio $\geq 5.0$, or when expression decreased, with a $Z$-score $\leq-2.0$ and a ratio of $\leq 0.2$. We selected 20 up-regulated and 20 down-regulated candidate genes whose function is known (Figure 5).

Table I shows that NNMT expression was 2,000 times higher in ACCS-LN-GFP than in ACCS-M-GFP cells. Similarly, the expression of synaptic vesicle glycoprotein 2B (SV2B) was also enhanced, though to a lower extent. Contrarily, the expression of gap junction protein alpha-1 (GJA1) and of Defensin B3 was attenuated to 0.003-fold. The expression of $\mathrm{N}$-cadherin, which is involved cell-cell 

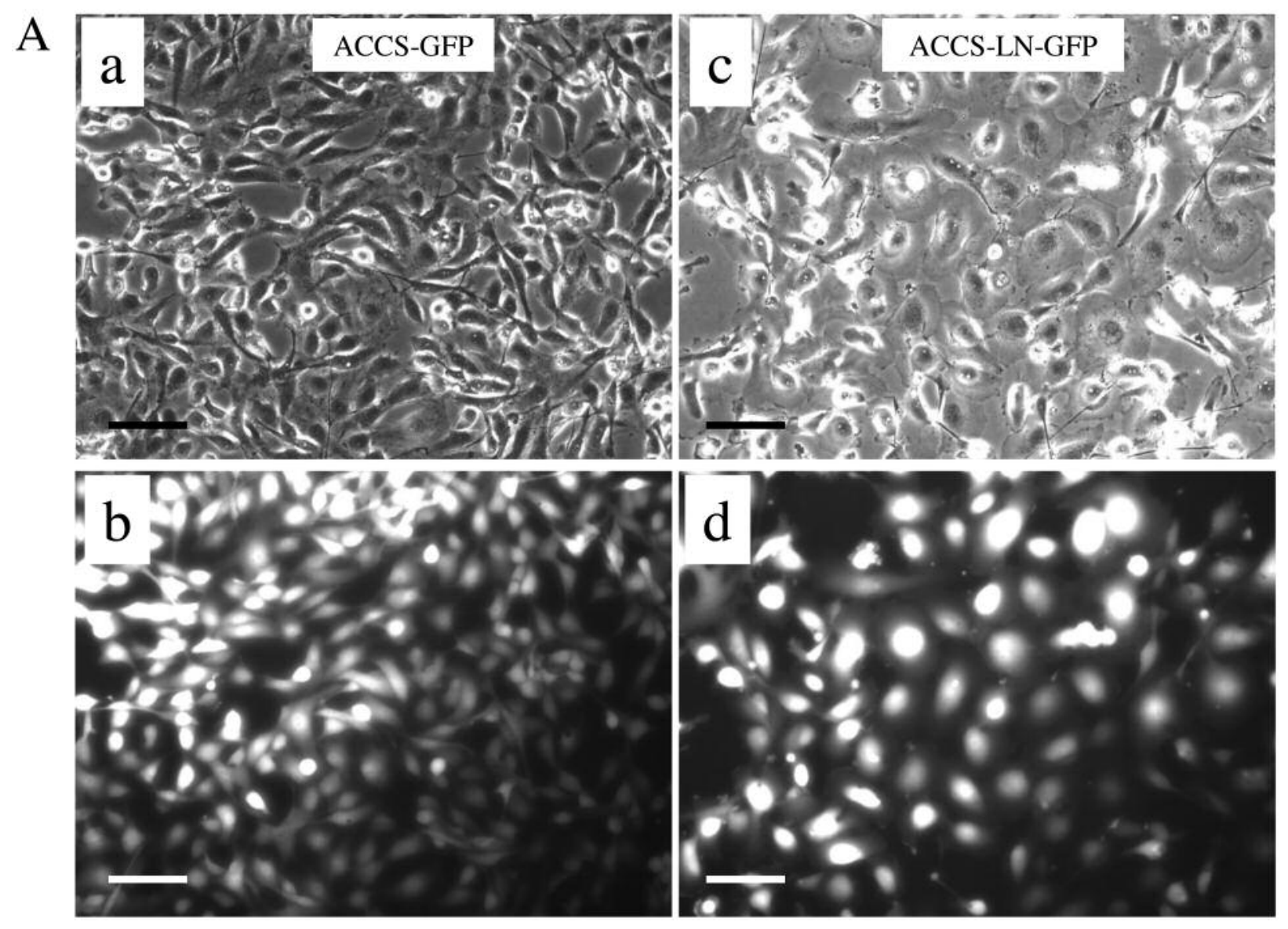

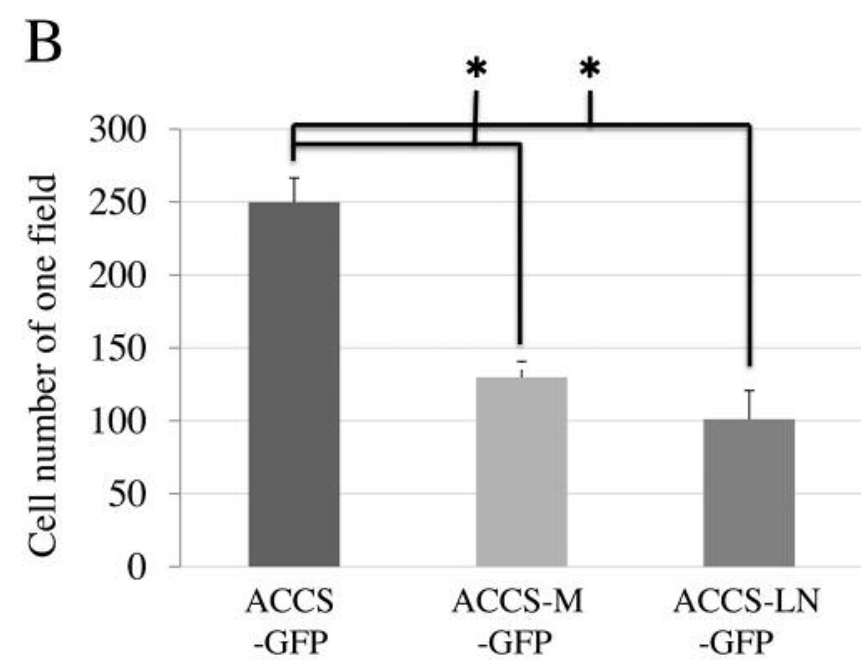

adhesion, was also attenuated in the ACCS-LN-GFP cells. No changes were observed in the expression of catenin or vimentin. Moreover, the expression of Brachyury, Snail and sex determining region Y-box 2 (SOX2), markers of cancer stem cells, did not significantly change (data not shown).
Changes in the expression of NNMT in the ACCS cell lines. The mRNA expression of NNMT in the ACCS cell lines was then investigated by qPCR. The expression level of $N N M T$ in ACCS-LN-GFP cells was same as that of ACCS-M-GFP (Figure 6A). Then, we examined the protein levels of NNMT 


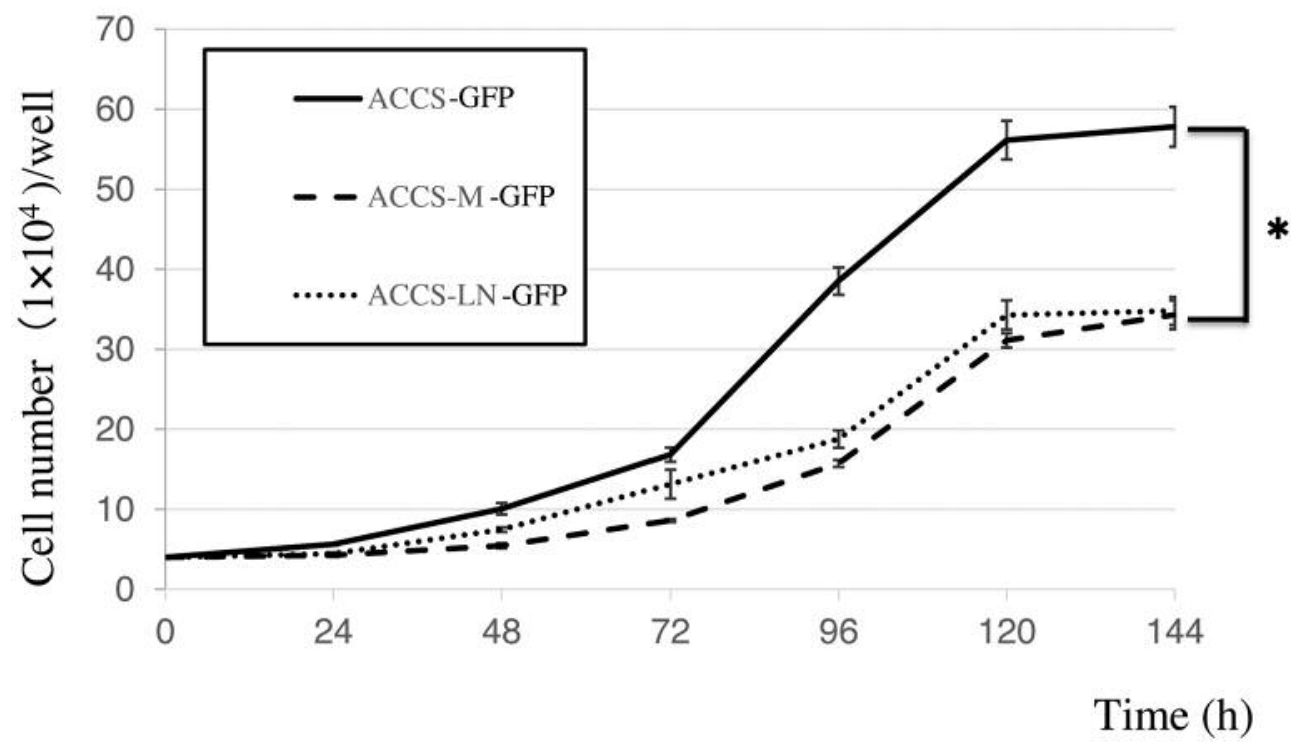

Figure 3. Cell growth for the ACCS cell lines evaluated by cell count. Assays were performed in triplicate and repeated three times. Each point represents the mean of three independent experiments with standard error. ${ }^{*} p<0.01$.

in our cell lines. ACCS-GFP cells expressed very low levels of NNMT, while the highly metastatic, and more so the lymph node metastatic cell lines, expressed higher NNMT levels (Figure 6B).

\section{Discussion}

AdCC is characterized by neural invasion and distant metastases, and shows low sensitivity to radiation and chemotherapy. Therefore, the effect of therapies is limited and the prognosis is generally poor $(9,10)$. We have previously studied the mechanism of invasion and metastasis in AdCC using in vitro AdCC invasion models $(11,12)$. However, metastasis is an intricate process that is difficult to mimic in vitro. Therefore, it was important to establish a spontaneous metastasis model for AdCC through orthotropic implantation. We previously established a highly-tumorigenic (ACCS-T-GFP) and a highly-metastatic (ACCS-M-GFP) cell line from the low-tumorigenicity cell line ACCS, using in vivo selection (5). These cell lines were polyclonal populations derived from the primary tumors. To identify metastasis-associated molecules, it is necessary to analyze the cells of the tumor metastases spontaneously originated. Published studies have investigated mechanism of metastasis in different kinds of carcinomas, through the injection of tumor cells into the mouse tail vein, and the subsequent establishment of metastatic cell lines $(12,13)$. However, these cell lines do not reflect key metastatic processes such as the detachment from the tumor site or the vascular invasion. Here, we generated a lymph node metastatic cell line (ACCS-LN-GFP) from ACCS cells. The present study is one of the few reports that established a cell line from metastatic tissue, isolated thanks to the fluorescence of the GFP the metastatic cells expressed $(14,15)$. Because of the way these cell lines were generated, they most likely represent a monoclonal cell population.

We compared the parental, the highly-metastatic cells and the lymph node metastatic cells. First, we examined the characteristics of the cell line generated. The proliferation of the cells with high metastatic potential was reduced compared to that of the parental cell line. However, their migration ability increased. This phenomenon is consistent with clinical observations in patients with AdCC.

We have reported that ACCS-M-GFP cells have a strong sphere-forming ability compared to ACCS-GFP cells (6). Similarly, the ACCS-LN-GFP cell line that we generated exhibited strong colonization capabilities, suggesting that ACCS-LN-GFP cells might have characteristics of CSC (data not shown).

In DNA microarray analysis, we compared the expression of markers of CSC such as Brachyury, Oct-4, SOX2, Snail, Slug and Twist in ACCS-M-GFP and ACCS-LN-GFP. The expression of these markers did not significantly change in the two cell lines. This observation does not deny the CSC potential of the ACCS-LN-GFP cells. We believe we did not find changes in the expression of CSC-related genes because ACCS-LN-GFP cells derive from ACCS-M-GFP cells, which, being metastatic, might already possess CSC features. 

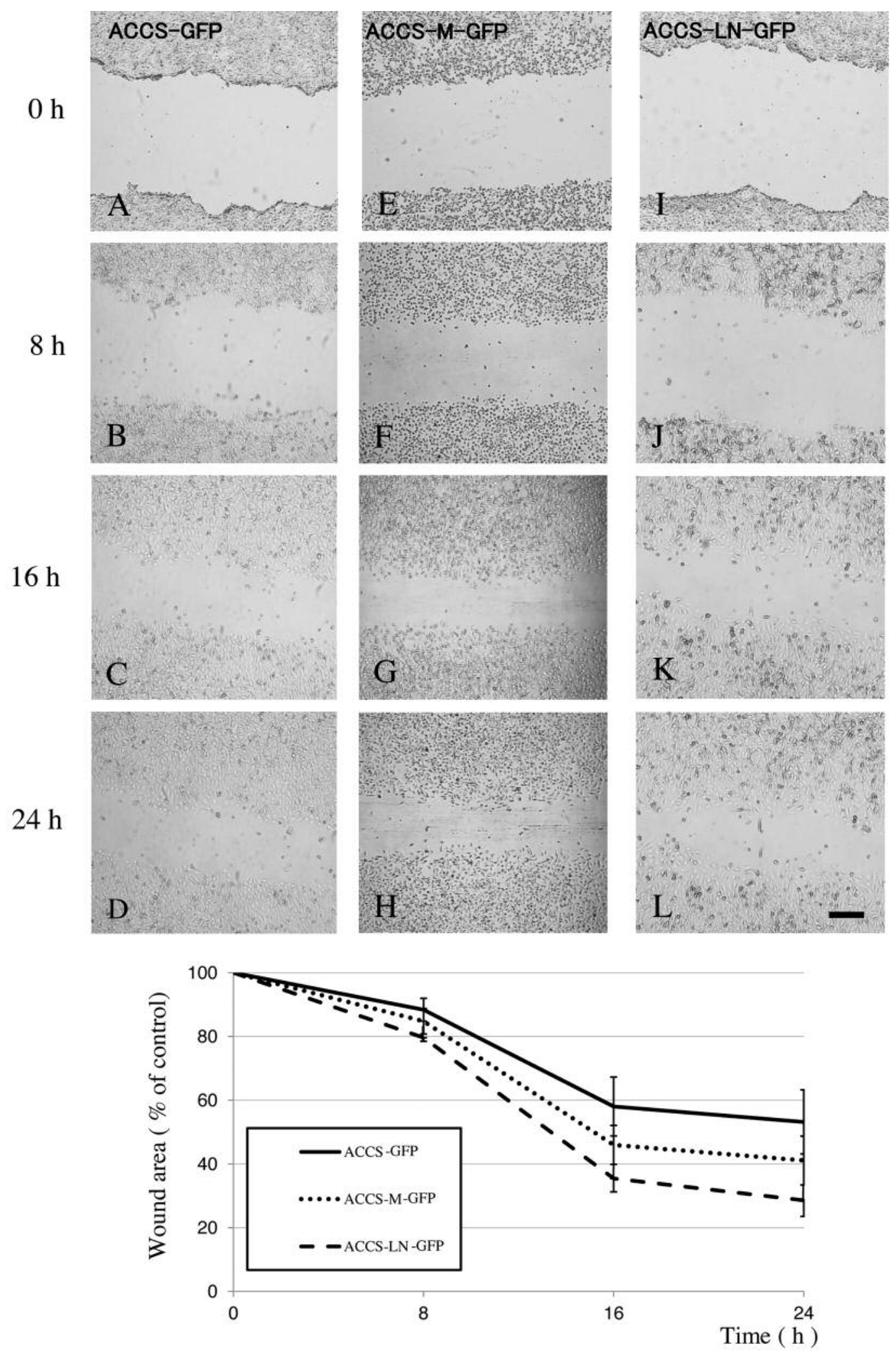

Figure 4. Cell migration in the ACCS cell lines. Migration was evaluated by using a wound-healing assay, as described in Materials and Methods. The wound regions were photographed $24 \mathrm{~h}$ after the start of the assay (A, E, I: $O \mathrm{~h} ; \mathrm{B}, \mathrm{F}, \mathrm{J}: 8 \mathrm{~h} ; \mathrm{C}, \mathrm{G}, \mathrm{K}: 16 \mathrm{~h} ; \mathrm{D}, \mathrm{H}, \mathrm{L}: 24 \mathrm{~h})$. (A-D) ACCS-GFP, (E-H) ACCS-M-GFP, (I-L) ACCS-LN-GFP. Data significance was analyzed using the Student's t-test: no significant differences were found. 


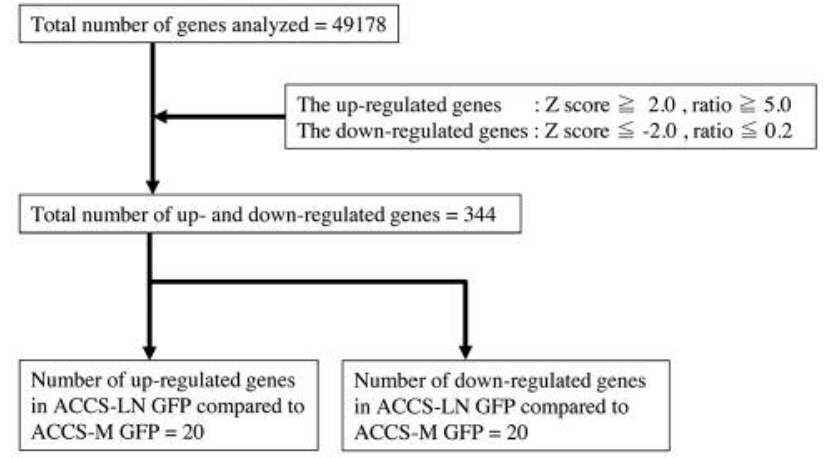

Figure 5. Screening of significant genes for cancer and metastasis. The 49,178 genes were filtered as indicated. Twenty up-regulated and 20 down-regulated candidate genes related to cancer or metastasis, and whose functions is known, were selected.

Decreased cell adhesion plays an important role in tumor metastasis. Our microarray data revealed the variation of fewer cell adhesion-related molecules than we expected. Among these, GJA1 was markedly down-regulated in ACCS-LN-GFP. Gap junctions are membrane channels composed of proteins called connexins. GJA1, also called connexin 43 , is the most ubiquitously expressed connexin (16) and is considered a tumor suppressor for its role in reversing the phenotype of cancer cells $(17,18)$. Decreased expression of connexin 43 remarkably diminishes the number of gap junctions, promoting cancer metastatic dissemination (19).

We also reported the loss of E-cadherin and integrins and the gain of vimentin in ACCS-M-GFP cells; this phenomenon suggested that the EMT is a putative event in AdCC metastasis (5). In the current study, the protein whose expression was changed the most in ACCS-LN-GFP cells was NNMT. NNMT is a recently identified potential tumor biomarker; it is a phase II metabolizing enzyme that catalyzes the $\mathrm{N}$-methylation of nicotinamide, pyridines, and other structural analogs involved in the biotic formation and in the detoxification of a number of xenobiotics $(20,21)$. Several studies have found that NNMT mRNA and protein levels are up-regulated and correlate with poor prognosis in several tumors including oral squamous cell carcinoma (22), renal cell cancer (23), lung cancer (24), prostate cancer (25), gastric carcinoma (26), and pancreatic cancer (27). In these studies, the expression of NNMT in cancerous tissues was higher than that in corresponding non-cancerous tissues. Moreover, the levels of NNMT correlated with tumor size, lymph node metastasis, distant metastasis, and overall survival. Furthermore, NNMT expression positively correlated with the phosphorylation of Akt, and negatively associated with disease-free survival and local recurrence-
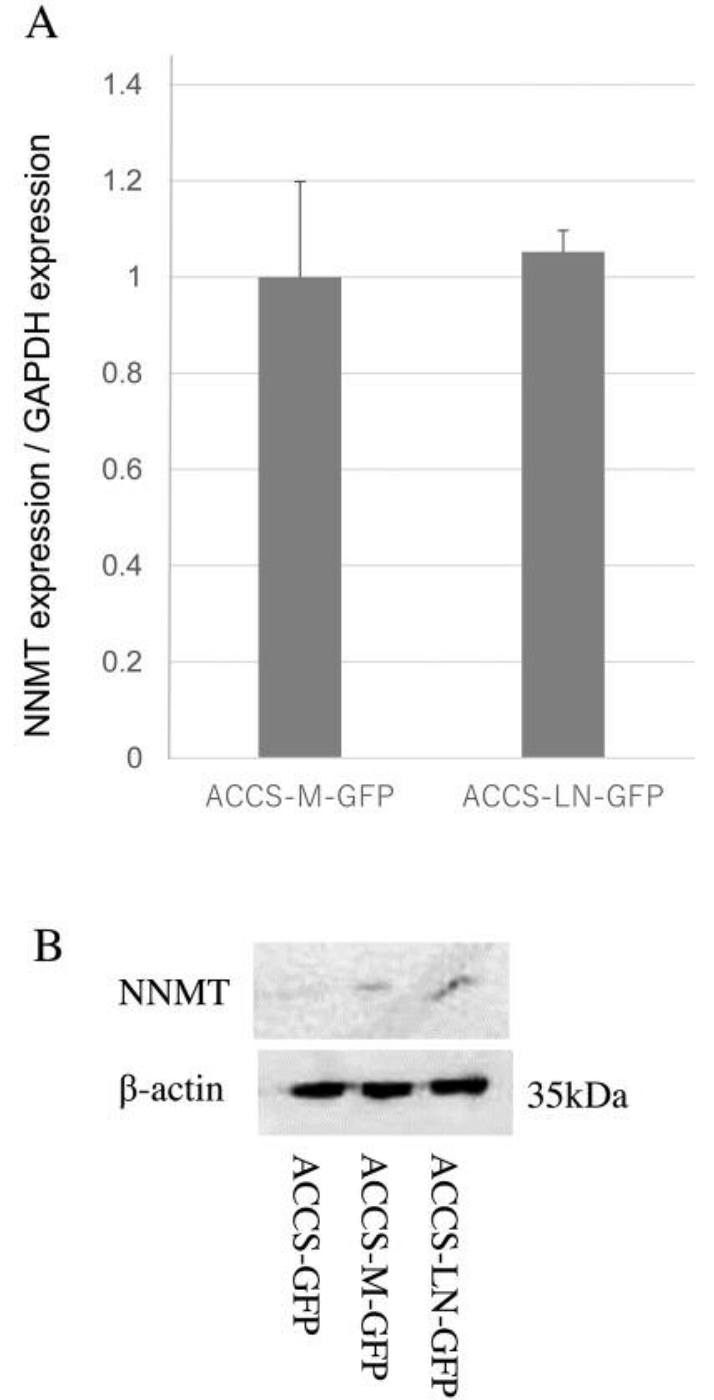

Figure 6. mRNA and protein levels of NNMT. (A) Relative levels of $m R N A$ related to various regulatory B subunits in ACCS-M-GFP and ACCS-LN-GFP cells, as determined by qPCR. Each bar represents the mean of five independent experiments with the standard error. $(B)$ Lymph node metastatic cells show increased expression of NNMT compared to the parental ACCS-M-GFP and ACCS-GFP cells. All ACCS cells were cultured for $24 \mathrm{~h}$ on culture dishes, prior to protein extraction. This experiment was performed in triplicate, and representative results are shown.

free survival (28). NNMT affects tumorigenesis because it impairs the methylation potential of cancer cells by consuming methyl units from S-adenosylmethionine to create the stable metabolic product 1-methylnicotinamide. As a result, NNMT-expressing cancer cells possess an altered epigenetic state that includes hypomethylated histones and other cancer-related proteins, combined with heightened 
Table I. The 20 most significant up- and down-regulated genes in lymph node metastatic ACCS-LN-GFP cells.

A. Up-regulated genes

\begin{tabular}{|c|c|c|c|c|c|c|}
\hline & ProbeID & GeneSymbol & GeneID & Description & Ratio & $\mathrm{Z}$ score \\
\hline 1 & A_23_P127584 & $N N M T$ & 4837 & Nicotinamide $\mathrm{N}$-methyltransferase & 2190.9 & 19.61 \\
\hline 2 & A_23_P100022 & $S V 2 B$ & 9899 & Synaptic vesicle glycoprotein $2 \mathrm{~B}$ & 226.2 & 9.59 \\
\hline 3 & A_23_P205164 & POU4F1 & 5457 & POU class 4 homeobox 1 & 110.2 & 8.32 \\
\hline 4 & A_33_P3228622 & OR14I1 & 401994 & Olfactory receptor, family 14 , subfamily I, member 1 & 100.7 & 8.16 \\
\hline 5 & A_24_P911906 & PCDH17 & 27253 & Protocadherin 17 & 48.9 & 3.47 \\
\hline 6 & A_33_P3300747 & $A D H F E 1$ & 137872 & Alcohol dehydrogenase, iron containing, 1 & 30.9 & 6.07 \\
\hline 7 & A_33_P3399150 & ORAOVI & 220064 & Oral cancer overexpressed 1 & 28.6 & 3.00 \\
\hline 8 & A_32_P140489 & GDF6 & 392255 & Growth differentiation factor 6 & 28.5 & 3.00 \\
\hline 9 & A_24_P189997 & PCSK6 & 5046 & Proprotein convertase subtilisin/kexin type 6 & 28.0 & 2.98 \\
\hline 10 & A_21_P0007483 & $S S P N$ & 8082 & Sarcospan & 27.3 & 2.96 \\
\hline 11 & A_33_P3292769 & NFAM1 & 150372 & NFAT activating protein with ITAM motif 1 & 26.8 & 2.95 \\
\hline 12 & A_33_P3249224 & $B A A T$ & 570 & Bile acid CoA:amino acid N-acyltransferase & 25.5 & 2.90 \\
\hline 13 & A_23_P258136 & MXRA5 & 25878 & Matrix-remodelling associated 5 & 24.2 & 2.86 \\
\hline 14 & A_32_P234518 & AWAT1 & 158833 & Acyl-CoA wax alcohol acyltransferase 1 & 24.0 & 2.85 \\
\hline 15 & A_23_P49759 & $C C L 1$ & 6346 & Chemokine (C-C motif) ligand 1 & 23.1 & 2.82 \\
\hline 16 & A_33_P3348569 & OR9G4 & 283189 & Olfactory receptor, family 9 , subfamily $\mathrm{G}$, member 4 & 21.3 & 2.75 \\
\hline 17 & A_19_P00315922 & WDFY4 & 57705 & WDFY family member 4 & 20.1 & 2.70 \\
\hline 18 & A_23_P103703 & $H S P B 7$ & 27129 & Heat shock protein family, member 7 & 18.4 & 2.62 \\
\hline 19 & A_33_P3384287 & $P A L M$ & 5064 & Paralemmin & 17.6 & 9.63 \\
\hline 20 & A_33_P3687198 & $H R K$ & 8739 & Harakiri, BCL2 interacting protein & 17.2 & 2.56 \\
\hline
\end{tabular}

B. Down-regulated genes

\begin{tabular}{|c|c|c|c|c|c|c|}
\hline & ProbeID & GeneSymbol & GeneID & Description & Ratio & $\mathrm{Z}$ score \\
\hline 1 & A_33_P3377090 & GJA1 & 2697 & Gap junction protein, alpha 1 & 0.0031 & -14.73 \\
\hline 2 & A_23_P169017 & $D E F B 103 B$ & 55894 & Defensin, beta 103B & 0.0037 & -14.28 \\
\hline 3 & A_33_P3241269 & CES1 & 1066 & Carboxylesterase 1 & 0.0049 & -9.43 \\
\hline 4 & A_33_P3332150 & APOBEC4 & 403314 & $\begin{array}{l}\text { Apolipoprotein B mRNA editing enzyme, } \\
\text { Catalytic polypeptide-like } 4\end{array}$ & 0.0090 & -8.35 \\
\hline 5 & A_33_P3260430 & $S P R R 2 A$ & 6700 & Small proline-rich protein $2 \mathrm{~A}$ & 0.0097 & -8.22 \\
\hline 6 & A_23_P161698 & $M M P 3$ & 4314 & Matrix metallopeptidase 3 & 0.0103 & -11.66 \\
\hline 7 & A_23_P210465 & $P I 3$ & 5266 & Peptidase inhibitor 3 & 0.0112 & -11.46 \\
\hline 8 & A_24_P190472 & $S L P I$ & 6590 & Secretory leukocyte peptidase inhibitor & 0.0125 & -11.18 \\
\hline 9 & A_24_P381199 & TRIM6 & 117854 & Tripartite motif containing 6 & 0.0156 & -7.37 \\
\hline 10 & A_24_P58204 & OR51B5 & 282763 & Olfactory receptor, family 51 , subfamily $\mathrm{B}$, member 5 & 0.0176 & -7.15 \\
\hline 11 & A_23_P1691 & $M M P 1$ & 4312 & Matrix metallopeptidase 1 & 0.0196 & -6.96 \\
\hline 12 & A_23_P2181 & $C Y B 5 R 2$ & 51700 & Cytochrome b5 reductase 2 & 0.0238 & -3.19 \\
\hline 13 & A_33_P3226761 & SOX18 & 54345 & SRY (sex determining region Y)-box 18 & 0.0250 & -6.54 \\
\hline 14 & A_23_P253536 & NPR3 & 4883 & Natriuretic peptide receptor 3 & 0.0265 & -3.09 \\
\hline 15 & A_19_P00811828 & ZNF737 & 100129842 & Zinc finger protein 737 & 0.0269 & -3.08 \\
\hline 16 & A_23_P129005 & NYNRIN & 57523 & NYN domain and retroviral integrase containing & 0.0274 & -6.38 \\
\hline 17 & A_23_P117851 & $C P L X 3$ & 594855 & Complexin 3 & 0.0283 & -3.04 \\
\hline 18 & A_23_P217379 & COL4A6 & 1288 & Collagen, type IV, alpha 6 & 0.0292 & -9.02 \\
\hline 19 & A_23_P64919 & $R E R G L$ & 79785 & RERG/RAS-like & 0.0383 & -2.77 \\
\hline 20 & A_23_P11644 & $S P R R 2 D$ & 6703 & Small proline-rich protein $2 \mathrm{D}$ & 0.0411 & -5.66 \\
\hline
\end{tabular}

expression of pro-tumorigenic gene products (29). Our data showed that the expression of NNMT enhanced tumor malignancy; in fact, the expression of NNMT increased from the low tumorigenic ACCS cell line to the highly-metastatic ACCS-M-GFP and lymph node metastasis ACCS-LN-GFP cell lines. These results suggest that NNMT might be a novel biomarker for malignancy. Accordingly, silencing of NNMT is associated to decreased cell proliferation and colony formation in vitro, and induces a marked reduction in tumor volume in vivo (30-32). These results suggest that inhibition of NNMT could represent a potential molecular approach for the treatment of malignant diseases. 
Some studies have found that NNMT is highly expressed in CSC $(33,34)$. As noted above, ACCS-LN-GFP cells might have CSC features, including the up-regulation of NNMT and the loss of GJA1, which lead to the EMT and consequent AdCC metastasis.

Recent reports indicate that NNMT can be detected in serum $(35,36)$, urine (37) and saliva (38), suggesting its usefulness as a diagnostic tool for AdCC and metastatic tumors in general. Importantly, recent studies indicate the microRNA in the serum may be useful for diagnosis or gene target therapy $(39,40)$. Hui et al. reported that NNMT levels correlate with the expression of microRNA-1291 in pancreatic carcinoma cells (41) and suggest that NNMT might be a marker of pancreatic carcinogenesis. Similarly, NNMT could serve as a biomarker for adenoid cystic carcinoma. Further studies are necessary to fully evaluate if NNMT can be used as a novel and effective tumor biomarker.

\section{Conflicts of Interest}

The Authors declare no financial or other potential conflict of interest in regard to this study.

\section{Acknowledgements}

This work was supported by JSPS KAKENHI Grant Number GAG5K20540 and 15K11257. The Authors gratefully acknowledge this financial support. The Authors would also like to thank Editage for English language editing.

\section{References}

1 Rapidis AD, Givalos N, Gakiopoulou H, Faratzis G, Stavrianos SD, Vilos GA, Douzinas EE and Patsouris E: Adenoid cystic carcinoma of the head and neck. Clinicopathological analysis of 23 patients and review of the literature. Oral Oncol 41: 328-335, 2005.

2 Ampil FL and Misra RP: Factors influencing survival of patients with adenoid cystic carcinoma of the salivary glands. J Oral Maxillofac Surg 45: 1005-1010, 1987.

3 Kobayashi Y, Sugiura T, Imajyo I, Shimoda M, Ishii K, Akimoto $\mathrm{N}$, Yoshihama $\mathrm{N}$ and Mori $\mathrm{Y}$ : Knockdown of the T-box transcription factor Brachyury increases sensitivity of adenoid cystic carcinoma cells to chemotherapy and radiation in vitro: Implications for a new therapeutic principle. Int J Oncol 44: 1107-1117, 2014

4 Hitre E, Budai B, Takácsi-Nagy Z, Rubovszky G, Tóth E, Remenár É, Polgár C and Láng I: Cetuximab and platinumbased chemoradio- or chemotherapy of patients with epidermal growth factor receptor expressing adenoid cystic carcinoma: a phase II trial. Br J Cancer 109: 1117-1122, 2013.

5 Ishii K, Shimoda M, Sugiura T, Seki K, Takahashi M, Abe M, Matsuki R, Inoue Y and Shirasuna K: Involvement of epithelialmesenchymal transition in adenoid cystic carcinoma metastasis. Int J Oncol 38: 921-931, 2011.

6 Shimoda M, Sugiura T, Imajyo I, Ishii K, Chigita S, Seki K, Kobayashi $\mathrm{Y}$ and Shirasuna K: The T-box transcription factor
Brachyury regulates epithelia-mesenchymal transitions in association with cancer stem-like cells in adenoid cystic carcinoma cells. BMC Cancer 12: 377, 2012.

7 Herrmann BG, Labeit S, Poustka A, King TR and Lehrach H: Cloning of the $\mathrm{T}$ gene required in mesoderm formation in the mouse. Nature 343: 617-622, 1990.

8 hirasuna K, Watatani K, Furusawa H, Saka M, Morioka S, Yoshioka $\mathrm{H}$ and Matsuya $\mathrm{T}$ : Biological characterization of pseudocyst-forming cell lines from human adenoid cystic carcinomas of minor salivary gland origin. Cancer Res 50: 41394145, 1990.

9 Ko JJ, Siever JE, Hao D, Simpson R, Lau HY: Adenoid cystic carcinoma of head and neck: clinical predictors of outcome from a Canadian centre. Curr Oncol 23: 26-33, 2016.

10 Ouyang DQ, Liang LZ, Zheng GS, Ke ZF, Weng DS, Yang WF, $\mathrm{Su}$ YX and Liao GQ: Risk factors and prognosis for salivary gland adenoid cystic carcinoma in southern china: A 25-year retrospective study Medicine (Baltimore) 96: e5964, 2017.

11 Abu-Ali S, Sugiura T, Takahashi M, Shiratsuchi T, Ikari T, Seki K, Hiraki A, Matsuki R and Shirasuna K: Expression of the urokinase receptor regulates focal adhesion assembly and cell migration in adenoid cystic carcinoma cells. J Cell Physiol 203: 410-419, 2005.

12 Seki K, Ishii K, Sugiura T, Takahashi M, Inoue Y and Shirasuna $\mathrm{K}$ : An adenoid cystic carcinoma cell line possessing high metastatic activity has high $\mathrm{NF}-\varkappa \mathrm{B}$ activation in response to TNF- $\alpha$. Oral Sci Int 2: 36-44, 2005.

13 Kuwabara Y, Yamada T, Yamada, Yamazaki K, Du WL, Kouji B, Aoki D and Sakamoto M: Establishment of an ovarian metastasis model and possible involvement of E-cadherin down-regulation in the metastasis. Cancer Sci 9: 1933-1939, 2008.

14 Su Y, Luo X, He BC, Wang Y, Chen L, Zuo GW, Liu B, Bi Y, Huang J, Zhu GH, He Y, Kang Q, Luo J, Shen J, Chen J, Jin X, Haydon RC, He TC and Luu HH: Establishment and characterization of a new highly metastatic human osteosarcoma cell line Clin Exp Metastasis 26: 599-610, 2009.

15 Eckhardt BL, Parker BS, van Laar RK, Restall CM, Natoli AL, Tavaria MD, Stanley KL, Sloan EK, Moseley JM and Anderson RL: Genomic analysis of a spontaneous model of breast cancer metastasis to 950 bone reveals a role for the extracellular matrix. Mol Cancer Res 3: 1-13, 2005.

16 Goodenough DA, Goliger JA and Paul DL: Connexins, Connexons, and intercellular communication (Review) Ann Rev Biochem 65: 475-502, 1996.

17 Tittarelli A, Guerrero I, Tempio F, Gleisner MA, Avalos I, Sabanegh S, Ortíz C, Michea L, López MN, Mendoza-Naranjo A and Salazar-Onfray F: Overexpression of connexin 43 reduces melanoma proliferative and metastatic capacity. Br J Cancer 113: 259-267, 2015.

18 Zhang A, Hitomi M, Bar-Shain N, Dalimov Z, Ellis L, Velpula KK, Fraizer GC, Gourdie RG and Lathia JD: Connexin 43 expression is associated with increased malignancy in prostate cancer cell lines and functions to promote migration. Oncotarget. 6: 11640-11651, 2015.

19 Mao XY, Li QQ, Gao YF, Zhou HH, Liu ZQ and Jin WL: Gap junction as an intercellular glue: Emerging roles in cancer EMT and metastasis. Cancer Lett 381: 133-137, 2016.

20 Aksoy S, Szumlanski CL and Weinshilboum RM: Human liver nicotinamide N-methyltransferase. J Bio Chem 269: 1483514840, 1994 
21 Hong S, Moreno-Navarrete JM, Wei X, Kikukawa Y, Tzameli I, Prasad D, Lee Y, Asara JM, Fernandez-Real JM, Maratos-Flier E and Pissios P: Nicotinamide N-methyltransferase regulates hepatic nutrient metabolism through Sirt1 protein stabilization. Nat Med 21: 887-894, 2015.

22 Sartini D, Santarelli A, Rossi V, Goteri G, Rubini C, Ciavarella D, Muzio L and Emanuelli M: Nicotinamide N-methyltransferase upregulation inversely correlates with lymph node metastasis in oral squamous cell carcinoma. Mol Med 13: 415421, 2007.

23 Zhang J, Xie XY, Yang SW, Wang J and He C: Nicotinamide Nmethyltransferase protein expression in renal cell cancer. $\mathrm{J}$ Zhejiang Univ Sci B 11: 136-143, 2010.

24 Sartini D, Morganti S, Guidi E, Rubini C, Zizzi A: Nicotinamide $\mathrm{N}$-methyltransferase in non-small cell lung cancer: Promising results for targeted anti-cancer therapy. Cell Biochem Biophys 67: 865-873, 2013.

25 Zhou W, Gui M, Zhu M, Long Z, Huang L, Zhou J, He L and Zhong L: Nicotinamide $\mathrm{N}$ methyltransferase is overexpressed in prostate cancer and correlates with prolonged progression free and overall survival time. Oncol Lett 8: 1175-1180, 2014.

26 Chen C, Wang X, Huang X, Yong H, Shen J, Tang Q, Zhu J, Ni $\mathrm{J}$ and Feng Z. Nicotinamide N-methyltransferase: a potential biomarker for worse prognosis in gastric carcinoma. Am J Cancer Res 6: 649-663, 2016.

27 Xu Y, Liu P, Zheng DH, Wu N, Zhu L, Xing C and Zhu J: Expression profile and prognostic value of NNMT in patients with pancreatic cancer Oncotarget 7: 19975-19981, 2016.

28 Win KT, Lee SW, Huang HY, Lin LC, Lin CY, Hsing CH, Chen LT and Li CF: Nicotinamide N-methyltransferase overexpression is associated with Akt phosphorylation and indicates worse prognosis in patients with nasopharyngeal carcinoma. Tumour Bio 34: 3923-3931, 2013.

29 Ulanovskaya OA, Zuhl AM, and Cravatt BF: NNMT promotes epigenetic remodeling in cancer by creating a metabolic methylation sink. Nat Chem Biol 9: 300-306, 2013.

30 Sartini D, Seta R, Pozzi V, Morganti S, Rubini C, Zizzi A, Tomasetti M, Santarelli L and Emanuelli M: Role of nicotinamide $\mathrm{N}$-methyltransferase in non-small cell lung cancer: in vitro effect of shRNA-mediated gene silencing on tumourigenicity. Biol Chem 396: 225-234, 2015.

31 Pozzi V, Sartini D, Morganti S, Giuliante R, Di Ruscio G, Santarelli A, Rocchetti R, Rubini C, Tomasetti M, Giannatempo G, Orlando F, Provinciali M, Lo Muzio L and Emanuelli M: RNA-mediated gene silencing of nicotinamide N-methyltransferase is associated with decreased tumorigenicity in human oral carcinoma cells. PLoS One 8: e71272, 2013.

32 Pozzi V, Mazzotta M, Lo Muzio L, Sartini D, Santarelli A, Renzi E, Rocchetti R, Tomasetti M, Ciavarella D and Emanuelli M: Inhibiting proliferation in $\mathrm{KB}$ cancer cells by RNA interferencemediated knockdown of nicotinamide $\mathrm{N}$-methyltransferase. Int J Immunopathol Pharmacol 24: 69-77, 2011.
33 Pozzi V, Sartini D, Rocchetti R, Santarelli A, Rubini C, Morganti S, Giuliante R, Calabrese S, Di Ruscio G, Orlando F, Provinciali M, Saccucci F, Lo Muzio L and Emanuelli M: Identification and characterization of cancer stem cells from head and neck squamous cell carcinoma cell lines. Cell Physiol Biochem 36: 784-798, 2015.

34 D'Andrea FP, Safwat A, Kassem M, Gautier L, Overgaard J and Horsman MR: Cancer stem cell overexpression of nicotinamide $\mathrm{N}$-methyltransferase enhances cellular radiation resistance. Radiother Oncol 99: 373-378, 2011.

35 Tomida M, Mikami I, Takeuchi S, Nishimura H, Akiyama H: Serum levels of nicotinamide N-methyltransferase in patients with lung cancer. J Cancer Res Clin Oncol 135: 1223-1229, 2009.

36 Roessler M, Rollinger W, Palme S, Hagmann ML, Berndt P, Engel AM, Schneidinger B, Pfeffer M, Andres H, Karl J, Bodenmüller H, Rüschoff J, Henkel T, Rohr G, Rossol S, Rösch $\mathrm{W}$, Langen $\mathrm{H}$, Zolg $\mathrm{W}$ and Tacke $\mathrm{M}$ : Identification of nicotinamide $\mathrm{N}$-methyltransferase as a novel serum tumor marker for colorectal cancer. Clin Cancer Res 11: 6550-6557, 2005.

37 Sartini D, Muzzonigro G, Milanese G, Pozzi V, Vici A, Morganti S, Rossi V, Mazzucchelli R, Montironi R and Emanuelli M: Upregulation of tissue and urinary nicotinamide $n$ methyltransferase in bladder cancer: Potential for the development of a urine-based diagnostic test cell. biochem biophys 65: 473-483, 2013.

38 Pozzi V, Sartini D, Morganti S, Giuliante R, Di Ruscio G, Santarelli A, Rocchetti R, Rubini C, Tomasetti M, Giannatempo G, Orlando F, Provinciali M, Lo Muzio L and Emanuelli M: RNA-Mediated gene silencing of nicotinamide Nmethyltransferase is associated with decreased tumorigenicity in human oral carcinoma cells. PLoS One 8: e71272, 2013.

39 Mine M, Yamaguchi K, Sugiura T, Chigita S, Yoshihama N, Yoshihama R, Hiyake N, Kobayashi Y and Mori Y: miR-203 Inhibits Frizzled-2 Expression via CD82/KAI1 Expression in Human Lung Carcinoma Cell.s PLoS One 10: e0131350, 2015.

40 Iorio MV and Croce CM: MicroRNA dysregulation in cancer: diagnostics, monitoring and therapeutics. A comprehensive review. EMBO Mol Med 4: 143-159, 2012.

41 Bi HC, Pan YZ, Qiu JX, Krausz KW, Li F, Johnson CH, Jiang $\mathrm{CT}$, Gonzalez FJ and Yu AM: N-methylnicotinamide and nicotinamide $\mathrm{N}$-methyltransferase are associated with microRNA-1291-altered pancreatic carcinoma cell metabolome and suppressed tumorigenesis. Carcinogenesis 35: 2264-2272, 2014.
Received October 6, 2017

Revised October 22, 2017 Accepted October 23, 2017 